It goes without saying that any number of the extremities of the intervals $\alpha_{i} b_{i}(i=0,1, \cdots, k)$ may lie at singular points provided that the conditions above stated for the point $e_{1}$ are satisfied at each of these points.

It should be noticed that the generalized Lamé's equation, whether looked at from Heine's* or from Klein's $\dagger$ standpoint, has as the exponents of each of its finite singular points the values $0, \frac{1}{2}$, so that in this case any or all of the intervals $a_{i} b_{i}$ may reach up to singular points.

We will note in conclusion that the cases we have just mentioned by no means exhaust the important applications of Theorems IV and V. For instance, the degenerate forms of Lamé's equation where two or more singular points coincide come immediately under Theorem IV.

HaRVARD University, CAMBridge, Mass.

\title{
THE CONSTRUCTION OF SPECIAL REGULAR RETICULATIONS ON A CLOSED SURFACE.
}

BY PROFLSSOR HENRY S. WHITE.

(Read in part at the Mathematical Conference in Chicago, January 1, 1897, and in final form at the Meeting of the American Mathematical Society, April 30, 1898.)

\section{INTRODUCTORY.}

The reticulations whose existence is here to be discussed are called regular because of two properties : the number of termini of edges assembled in one vertex is the same for all vertices of the reticulation, and the number of edges in the boundary of a face is the same for all faces. These two numbers, $r$ and $s$, together with $p$, the deficiency of the supporting surface, shall be assumed to characterize the reticulation sufficiently for present purposes. Of regular reticulations classified on this basis, only a finite number of classes are possible on a surface of given deficiency. Some of these possible classes, if $p>2$, are derivable from those of lower deficiency; those not so derivable are properly

* Handbuch der Kugelfunctionen, vol. I., p. 445.

†Cf. my book: "Ueber die Keihenentwickelungen der Potentialtheorie," p. 114.

$\ddagger$ It is in fact easy to see that they may turn back at the singular points and thus cover parts of the $x$-axis more than once. Cf. p. 123 of my book just referred to. 
called special or primitive. In a paper * read at the Buffalo meeting of the American Mathematical Societry I explained this distinction, and showed tables of all primitive regular reticulations for deficiencies 2 and 3 . Those tables had only a restrictive, not a positive significance ; and fortunately I was able to show also sets of models proving empirically the existence of types of all classes admitted by the tables.

Further study shows that there are certain connections among the tabulated numbers, rendering probable the derivation of types of all the classes belonging to the same surface from a single fundamental reticulation, provided that the deficiency of the surface is lower than 9. Professor Ellery W. Davis has demonstrated the occurrence of exceptional pairs of characteristics $r, s$, devoid of such connection, and has thus shown critically one of the limitations of the present discussion. Excluding such Davis reticulations, we find our problem reduced to that of the construction of one fundamental reticulation for each surface. This can always be effected, by a method due to Riemann, the so-called canonical dissection of a Riemann surface, with certain obviously admissible modifications. The method allows, moreover, an examination of the grouping of the several faces about each vertex ; or dually, of the vertices about each face. While it is not proposed here to develop these minutiæ, it is desired to give a working outline of three essential parts of the systematic verification of each table of admissible reticulations :

1. The mutual derivations of two dual reticulations from each other ;

2. Two (dual) processes for multiplying the number of vertices or faces ;

3. The dissection of a Riemann surface into a fundamental polygon.

To obviate confusion it should be understood that the regularity of these reticulations is not the same as that defined by Dyck and Klein in function-theoretic investigations ; the two definitions overlap, but neither includes the other.

\section{§1. Duality.}

Two reticulations are dual to each other when the numbers $r, s, V, F$, of the first are equal respectively to $s^{\prime}, r^{\prime}$, $F^{\prime}, V^{\prime}$, of the second; and when each face of the one corre-

\footnotetext{
*Printed in Bulletin for December, 1896, pp. 116-121.
} 
sponds to a vertex of the other in such a way that the succession of vertices about each face corresponds exactly to the succession of faces about the corresponding vertex. From this definition it is clear that if one of two dual reticulations is regular, the other must be so. Either may be derived from the other, so far as its numerical or tactical character is concerned, in the following way : In each face of the first, assume a point for a vertex of the second. On every edge of the first, mark a point where an edge of the second shall cross it. Connect each new vertex to the designated points on all edges of the face in which it lies, and consider as one continuous line every pair of these join-lines which meet at an edge of the first reticulation. Then the new vertices and lines will constitute a second reticulation dual to the first. For the numbers $r$ and $s$ of the first are by construction equal to $s^{\prime}$ and $r^{\prime}$ of the second, likewise $V$ and $F$ to $F^{\prime}$ and $V^{\prime}$ respectively. And since two faces in contact along an edge are replaced by two vertices terminating a new edge, and vice versa, therefore the order of succession about each face and about each vertex has been strictly preserved, as the definition requires.

The same freedom to substitute one of two dual figures for the other, which is allowed in projective geometry, is therefore allowed equally in every purely topological study of reticulations. Of practical importance is this observation only when, of two dual reticulations whose existence is to be proved, the one is somewhat the easier for the intuition to grasp.

\section{§2. Multiplying the Number of Faces or of Vertices.}

If a regular reticulation is given, and the tables show as possible a second which has the same number of vertices, while the number of faces is an exact multiple of that in the first, the obvious way of deriving the second from the first is by drawing diagonals from vertex to vertex as often as necessary. This does not change the order of sequence among faces already meeting at any vertex, but inserts one new face with every diagonal drawn from that vertex. In each face, the initial point of each required diagonal may be arbitrary, and hence a comparatively large number of alternatives may have to be tried before finding one that satisfies the obvious restriction : that all vertices are to become termini of the same number of diagonals. A finite number of trials will always prove whether it is possible to effect the proposed modification upon a reticulation of given type.

Reversing now the significance of the numbers, suppose 
that a second reticulation is to be derived from the first by retaining the number of faces but multiplying the number of its vertices. For this purpose the edges may be thought of as elastic fibres, the termini as loops or eyelets sliding freely upon all other edges. At each vertex of the given reticulation the termini are to be divided into groups of suitable size-the extremes larger by one than the intermediate groups ; and each group is thought of as sliding away from its next preceding neighboring group along some edge apportioned to it. The point where it rests becomes a new terminus. Otherwise the termini at a vertex may be regarded as clustered along a small but extensible circle, which is to be cut at some selected point and stretched until the segments of arc between clusters become edges of finite length. Each such intercalated edge augments by one the number of boundaries of two faces; and the restriction is like that imposed a moment ago upon the dual operation, that all faces must have the number of their edges augmented alike.

Tables of Special Regular Reticulation-numbers.

\begin{tabular}{|c|c|c|c|c|}
\hline Deficiency : & \begin{tabular}{|cr|rr}
\multicolumn{2}{|c|}{4,} & \multicolumn{2}{c}{5,} \\
$1_{16}$ & $1_{16}$ & $1_{20}$ & $1_{20}$ \\
$1_{18}$ & $2_{9}$ & $1_{22}$ & $2_{11}$ \\
$1_{28}$ & $7_{4}$ & $1_{24}$ & 3 \\
2 & $2_{8}$ & $1_{10}$ \\
$2_{10}$ & $4_{10}$ & $1_{30}$ \\
$2_{12}$ & $4_{6}$ & $1_{36}$ & $9_{4}$ \\
$2_{16}$ & $8_{4}$ & $1_{54}$ & $18_{3}$ \\
$2_{24}$ & $16_{3}$ & $2_{15}$ & $5_{6}$ \\
$4_{7}$ & $4_{7}$ & $3_{22}$ & $22_{3}$ \\
$4_{10}$ & $10_{4}$ & $6_{7}$ & $7_{6}$ \\
$4_{15}$ & $20_{3}$ & & \\
$8_{7}$ & $14_{4}$ &
\end{tabular} & \begin{tabular}{rr}
\multicolumn{2}{c}{6,} \\
$1_{24}$ & $1_{24}$ \\
$1_{26}$ & $2_{13}$ \\
$1_{44}$ & $11_{4}$ \\
$1_{66}$ & $22_{3}$ \\
$2_{14}$ & $2_{14}$ \\
$2_{15}$ & $3_{10}$ \\
$2_{16}$ & $4_{8}$ \\
$2_{18}$ & $6_{6}$ \\
$2_{20}$ & $8_{5}$ \\
$2_{24}$ & $12_{4}$ \\
$2_{36}$ & $24_{3}$ \\
$3_{26}$ & $26_{3}$ \\
$4_{9}$ & $4_{9}$ \\
$4_{14}$ & $14_{4}$ \\
$4_{21}$ & $28_{3}$ \\
$6_{8}$ & $8_{6}$ \\
$6_{16}$ & $32_{3}$ \\
$8_{9}$ & $18_{4}$ \\
$12_{11}$ & $44_{3}$
\end{tabular} & $\begin{array}{rr} & 7, \\
\mathrm{I}_{28} & 1_{28} \\
\mathrm{I}_{30} & 2_{15} \\
\mathrm{I}_{52} & 13_{4} \\
\mathrm{l}_{78} & 26_{3} \\
2_{21} & 7_{6}\end{array}$ & \begin{tabular}{cc}
\multicolumn{2}{c}{8,} \\
$1_{32}$ & $1_{32}$ \\
$1_{34}$ & $2_{17}$ \\
$1_{36}$ & $3_{12}$ \\
$1_{40}$ & $5_{8}$ \\
$1_{42}$ & $6_{7}$ \\
$1_{50}$ & $10_{5}$ \\
$1_{60}$ & $15_{4}$ \\
$1_{90}$ & $30_{3}$ \\
$2_{18}$ & $2_{18}$ \\
$2_{20}$ & $4_{10}$ \\
$2_{24}$ & $8_{6}$ \\
$2_{32}$ & $16_{4}$ \\
$2_{48}$ & $32_{3}$ \\
$3_{34}$ & $34_{3}$ \\
$4_{11}$ & $44_{11}$ \\
$4_{12}$ & $6_{8}$ \\
$4_{15}$ & $12_{5}$ \\
$4_{18}$ & $18_{4}$ \\
$4_{27}$ & $36_{3}$ \\
$6_{10}$ & $10_{6}$ \\
$6_{20}$ & $40_{3}$ \\
$8_{11}$ & $22_{4}$ \\
$10_{8}$ & $16_{5}$ \\
$12_{13}$ & $52_{3}$
\end{tabular} \\
\hline
\end{tabular}


These processes may suffice for verifying empirically any given restrictive table if in the first place a fundamental polygon on the surface is given, and if in the second place the table shows each pair of values, $V$ and $F$, to have one value in common with a smaller pair. This latter condition is fulfilled in the tables previously published for $p=2$ and $p=3$, also in the summary on the preceding page for $p=4,5,6,7,8$, but not for $p=9$. Each set of numbers belonging to one reticulation is given in the order $F_{s} V_{r}$. The last two in column 9 are specials of the Davis type.

\section{§3. Dissection of a Riemand Surface Yields a Funda- mental Polygon.}

Any finite closed surface may be regarded as an ideal Riemann surface. Disregarding its historical relation to the theory of functions, we wish to avail ourselves of a well-known method belonging strictly to topology or analysis situs, for laying out circuits or lines of section which shall reduce a multiply-connected surface to a simply-connected one, with a continuous boundary. If the deficiency of the surface is $p$, that means that exactly $p$ circuits can be drawn in it which shall be non-collapsible (irreducible) and non-intersecting, and which shall not divide the surface into separate portions. After that, $p$ conjugate circuits can be drawn, each of which shall be non-collapsible, and shall meet one, and only one, of the former circuits, and that in but a single point; these circuits also leave the surface still one connected whole. If now lines be drawn, connecting in series all $p$ points where pairs of conjugate circuits intersect, the dissection is complete, and the resulting surface is simply-connected with a single continuous boundary, consisting of all the circuits and join-lines, each traversed twice in opposite senses.

For our use, a better statement of the theorem is this: Through any point in the surface $2 p$ non-collapsible circuits can be drawn, each of which shall be cut in that point by at least one other, and which shall not intersect elsewhere. Such $2 p$ circuits cut the surface into one simply-connected polygon.

This is evidently a fundamental polygon such as we require. It has 1 vertex and $2 p$ edges. In the vertex are $4 p$ termini, and its boundary has $4 p$ consecutive edges. Comparing this with the formulæ

$$
V=\frac{(4 p-4) \cdot s}{(r-2)(s-2)-4}, \quad F=\frac{(4 p-4) \cdot r}{(r-2)(s-2)-4},
$$


we see that $r=s=4 p$ will give the values $V=1, F=1$, as expected. We conclude: There is therefore on every closed (two-sided) surface of deficiency $p$ a fundamental polygon of one vertex and having $4 p$ edges united in pairs.

It is now an easy matter, by either of the two processes described above, to cut this polygon by diagonals or intercalated edges, into as many faces with one vertex, or vertices upon one face, as there are factors in $4 p-2$. For if we set in the formulæ cited above :

$$
\begin{gathered}
4 p-2=g(s-2), \\
4 p+2 g-4=r-2,
\end{gathered}
$$

we shall find $V=1, F=g=$ any factor of $4 p-2$.

This establishes the existence of all tabulated reticulations having either $V$ or $F=1$. For others, apparently derivable from these, the verification needs in each case only a finite number of trials. There would arise further theoretical questions, of whose solution experiment may be expected to furnish some suggestion. Before leaving this topic, it is worth while to add a remark, which in many trials leads to the highest attainable degree of symmetry - a remark upon the arrangement of edges about the vertex of the fundamental polygon.

The order of the edges about the vertex of the fundamental polygon, or any derivative of it, is arbitrary, under the sole restriction that between the opposite termini of each several edge there shall occur a terminus of at least one edge, whose other terminus does not occur in the same interval, and all termini must be divisible thus, in at least one way, into mutually exclusive sets of four. In other words, in the succession of termini about the afore-mentioned vertex, each pair of termini belonging to the same edge must be separated by the two termini of at least one other edge. This restriction, it will be seen, is always satisfied by arranging in succession one terminus from each of the edges, followed by the opposite termini in the same order. Thus each circuit is cut by all the others ; this renders also the fundamental polygon dual in type to itself.

After so much of description of the processes employed, it is easy for any one to formulate a notation which shall exhibit at each stage of the derivation process :

1. A definite mark for each edge, this mark to remain the same through all stages after its introduction ;

2. A mark for each vertex, and a list of the edges and faces in their order of grouping about each vertex ; 
3. A mark for each face, and a list of the edges and vertices in their order upon the boundary of each face.

Such a notation must contain a mark of distinction for the two sides of an edge; an easy matter if the direction of positive rotation be adopted uniformly in listing arrangements about the vertices and faces respectively.

These processes, and the proved existence of fundamental polygons, open a range of particular problems of considerable interest. But of even superior interest must be, at least until it is solved, the problem of finding a method for constructing, a priori, upon a given surface the exceptional (Davis) special reticulations whose characteristics are given by the restrictive tables.

NORTH WESTERN UNIVERSTTY, April, 1898.

\section{SYSTEMS OF SIMPLE GROUPS DERIVED FROM THE ORTHOGONAL GROUP.}

BY DR. L. E. DICKSON.

1. In the February number of the Bulletrs I determined the order $\omega$ of the group $G$ of orthogonal substitutions of determinant unity on $m$ indices in the $G F\left[p^{n}\right]$ and proved that, for $* p^{n}>5, p \neq 2$, the group is generated by the substitutions

$$
O_{i, j}^{\alpha, \beta}: \quad \begin{aligned}
& \xi_{i}^{\prime}=\alpha \xi_{i}+\beta \xi_{j}, \\
& \xi_{j}^{\prime}=-\beta \xi_{i}+\alpha \xi_{j},
\end{aligned} \quad\left(\alpha^{2}+\beta^{2}=1\right) .
$$

The structure of $G$ was determined for the case $p=2$. I have since proved $\dagger$ that for every $m>4$ and every $p^{n}>5$ of the form $8 l+3$ or $8 l+5$, the factors of composition of $G$

* The fact that $p^{n}=3$ is an exception was not pointed out in the BULLETIN. In fact Jordan hall not proven case $2^{\circ}$ of $\& 211$ when $-1=$ square, so that the case $a^{2}=b^{2}=c^{2}=\ldots=1$ was unsolved when $p=3$, $m=3 k+1$. The theorem is readily proven when $p^{n}=3^{n}, n>1$; but for $p^{n}=3$ an additional generator is necessary and sufficient, viz.,

$$
W=\left(\begin{array}{llll}
1 & 2 & 2 & 2 \\
1 & 2 & 1 & 1 \\
1 & 1 & 2 & 1 \\
1 & 1 & 1 & 2
\end{array}\right), W^{3}=1
$$

$\dagger$ A preliminary account was presented before the Mathematical Conference at Chicago, December 30, 1897. 